\title{
Expression of Arabidopsis FCS-Like Zinc finger genes is differentially regulated by sugars, cellular energy level, and abiotic stress
}

\section{OPEN ACCESS}

Edited by:

Girdhar Kumar Pandey,

University of Delhi, India

Reviewed by:

Frederik Börnke,

Leibniz Institute of Vegetable and Ornamental Crops, Germany

Manu Agarwal,

University of Delhi, India

*Correspondence:

Ashverya Laxmi,

National Institute of Plant Genome Research, Aruna Asaf Ali Road, New Delhi-110067, India

ashverya_laxmi@nipgr.ac.in

Specialty section: This article was submitted to Plant Physiology, a section of the journal Frontiers in Plant Science

Received: 04 June 2015

Accepted: 31 August 2015

Published: 24 September 2015

Citation:

Jamsheer KM and Laxmi A (2015) Expression of Arabidopsis FCS-Like

Zinc finger genes is differentially regulated by sugars, cellular energy

level, and abiotic stress.

Front. Plant Sci. 6:746.

doi: 10.3389/fpls.2015.00746

\section{Muhammed Jamsheer K and Ashverya Laxmi*}

National Institute of Plant Genome Research, New Delhi, India

Cellular energy status is an important regulator of plant growth, development, and stress mitigation. Environmental stresses ultimately lead to energy deficit in the cell which activates the SNF1-RELATED KINASE 1 (SnRK1) signaling cascade which eventually triggering a massive reprogramming of transcription to enable the plant to survive under low-energy conditions. The role of Arabidopsis thaliana FCS-Like Zinc finger (FLZ) gene family in energy and stress signaling is recently come to highlight after their interaction with kinase subunits of SnRK1 were identified. In a detailed expression analysis in different sugars, energy starvation, and replenishment series, we identified that the expression of most of the $F L Z$ genes is differentially modulated by cellular energy level. It was found that $F L Z$ gene family contains genes which are both positively and negatively regulated by energy deficit as well as energy-rich conditions. Genetic and pharmacological studies identified the role of HEXOKINASE 1- dependent and energy signaling pathways in the sugar-induced expression of $F L Z$ genes. Further, these genes were also found to be highly responsive to different stresses as well as abscisic acid. In over-expression of kinase subunit of $S n R K 1, F L Z$ genes were found to be differentially regulated in accordance with their response toward energy fluctuation suggesting that these genes may work downstream to the established SnRK1 signaling under lowenergy stress. Taken together, the present study provides a conceptual framework for further studies related to SnRK1-FLZ interaction in relation to sugar and energy signaling and stress response.

Keywords: Arabidopsis, FLZ gene family, sugar signaling, energy signaling, SnRK1, low-energy stress, abiotic stress, HXK1

\section{Introduction}

Life is modulated by an array of internal and external factors which act as signals to control the fate of the organisms. In response to these factors, organisms tone their growth and reproduction so that they will effectively survive and reproduce. Cellular energy status is directly dependent on the supply of sugars. Internal sugar status is an important factor which controls the lifespan and aging in metazoans, yeast and plants (Heilbronn and Ravussin, 2005; Minina et al., 2013). Adequate sugar supply is required for the better stress tolerance. Glucose regulates a large number of genes involved in both biotic and abiotic stress response (Price et al., 2004). Sucrose promotes the survival 
of plants under salt stress through G-protein signaling (Colaneri et al., 2014). Many of the mutants defective in sugar responses were found to be allelic to mutants of abscisic acid (ABA) and ethylene signaling suggesting a large interplay between sugar and stress signaling (Leon and Sheen, 2003).

In plants, stressful environment ultimately leads to energy deprivation in the cell which activates the evolutionarily conserved multi-subunit serine-threonine kinase SNF1RELATED KINASE 1 (SnRK1). SnRK1 acts a central integrator of energy metabolism, stress, and developmental cues (BaenaGonzález et al., 2007). Similar to its mammalian homolog AMP-activated protein kinase (AMPK), SnRK1 was also found to be phosphorylated on its T-loop and its dephosphorylation is inhibited by 5'-AMP (Sugden et al., 1999; Hey et al., 2007). In response to low-energy stress, SnRK1 promotes catabolism and minimize anabolism through reprogramming the transcriptional machinery which enables the plants to survive under lowenergy conditions (Baena-González et al., 2007). It was also found that SnRK1 imparts tolerance to various biotic as well as abiotic stresses via controlling the transcription of genes involved in stress tolerance (Baena-González et al., 2007; Baena-González and Sheen, 2008; Cho et al., 2012; Confraria et al., 2013). SnRK1 activity is essential for establishing flooding stress tolerance in rice (Cho et al., 2012). A PP2C hub is involved in the regulation of both SnRK1 and ABA signaling suggesting an integrated molecular pathway in the mitigation of low-energy condition and abiotic stresses (Rodrigues et al., 2013).

The plant-specific FCS-like zinc finger (FLZ) domain proteins are implicated in the regulation of various biotic and abiotic stresses. FCS-LIKE ZINC FINGER 9 /MEDIATOR OF ABAREGULATED DORMANCY 1 (FLZ9/MARD1) is found to be involved in the ABA-mediated seed dormancy and up-regulated during leaf senescence (He et al., 2001; He and Gan, 2004). Over-expression of FCS-LIKE ZINC FINGER 4 /INCREASED RESISTANCE TO MYZUS PERSICAE 1 (FLZ4/IRM1) rendered plants shorter which creates mechanical resistance to aphid attack (Chen et al., 2013). Over-expression of a wheat FLZ gene, saltrelated hypothetical protein (TaSRHP) in Arabidopsis thaliana resulted in enhanced resistance to salt and drought stress (Hou et al., 2013). The expression analysis from the publically available microarray data suggests that members of $A$. thaliana FLZ gene family is responsive to ABA, JA, various abiotic stresses, glucose, and nitrogen and phosphorous deficiency (Nietzsche et al., 2014). Protein-protein interaction studies identified that all $18 \mathrm{~A}$. thaliana FLZ proteins interact with kinase subunits of SnRK1 (Arabidopsis Interactome Mapping Consortium, 2011; Nietzsche et al., 2014).

The available evidence indicates the possible role of FLZ gene family in stress tolerance and adaptive growth. Similarly, their physical interaction with kinase subunits of SnRK1 suggests their relation with SnRK1 signaling. However, their relation with SnRK1 signaling particularly during energy fluctuations in the cell and abiotic stresses is not explored yet. In this study, we analyzed the transcriptional regulation of FLZ gene family during low-energy stress and energy rich conditions. We also identified that different sugar signaling pathways regulate the sugar-dependent transcription of FLZ genes. We also analyzed the expression of these genes under ABA treatment and different stresses with particular emphasize on salt stress. Over-expression of kinase subunit of SnRK1 resulted in differential regulation of some of the FLZ genes suggesting that SnRK1 signaling transcriptionally regulates these genes in the plants.

\section{Materials and Methods}

\section{Plant Material and Growth Conditions}

The A. thaliana Columbia (Col-0) and Landsberg erecta (Ler) ecotypes were used as controls in the experiments. All the experiments were done in Col-0 unless stated. Seeds of gin2-1 (CS6383) were obtained from ABRC (https://abrc.osu.edu; Moore et al., 2003). KIN10 OE2 seeds were provided by Prof. Filip Rolland (Metabolic signaling group, KU Leuven, Baena-González et al., 2007). For all experiments, seeds were surface sterilized and stored at $4^{\circ} \mathrm{C}$ for $48 \mathrm{~h}$ in dark for stratification. The imbibed seeds were grown on square petri plates containing 0.5X MS medium with $1 \%$ sucrose and $0.8 \%$ agar. The plates were kept vertically for germination and growth in climate-controlled growth room under $16: 8 \mathrm{~h}$ photoperiod with $22 \pm 2^{\circ} \mathrm{C}$ temperatures and $60 \mu \mathrm{mol} \mathrm{m} \mathrm{m}^{-2} \mathrm{~s}^{-1}$ light intensity unless stated. Five-days old seedlings grown in standard growth conditions were used for all experiments and at least 40 seedlings were harvested for each sample. KIN1OE2 and Ler seedlings grown for 5 days in the standard growth conditions were used for gene expression analysis.

\section{Sugar Starvation and Replenishment Assay}

Five-days old uniformly grown Col-0 seedlings under standard growth condition were used for sugar starvation and replenishment assay. Plants were starved in 0.5X MS liquid medium without sucrose in $22^{\circ} \mathrm{C}$ at $140 \mathrm{rpm}$ in darkness. Samples were collected after 3, 6, 12, and $24 \mathrm{~h}$ time points of starvation. After $24 \mathrm{~h}$ time point, the plants were transferred to $0.5 \mathrm{X}$ liquid medium with sucrose and grown under $22^{\circ} \mathrm{C}$ at $140 \mathrm{rpm}$ in the light. Samples were collected after 3, 6, 12, and $24 \mathrm{~h}$ time points of replenishment.

\section{Sugar Sensitivity Assays and Treatment with Chemical Inhibitors}

The sugar sensitivity assay is done as described previously using $3 \%$ glucose/sucrose/3-O-methylglucose/mannose/ mannitol individually (Mishra et al., 2009). For sugar sensitivity assay in glucose signaling mutant gin2-1, the Ler and gin2-1 seedlings were subjected to the same treatment. For sugar sensitivity assay along with metabolic inhibitors, Col-0 seedling were transferred to $0.5 \mathrm{X}$ MS medium with $3 \%$ glucose or sucrose with 3\% 2-Deoxy-D-glucose (2DG)/5 $\mu \mathrm{M}$ Antimycin-A (AmA)/50 $\mu \mathrm{M}$ 2,4-Dinitrophenol (DNP)/10 $\mu \mathrm{M}$ Carbonyl cyanide $m$-chlorophenyl hydrazone (CCCP) (Sigma-Aldrich) individually in the same treatment condition mentioned above. For 2DG treatment, 5-days old Col-0 seedlings grown in standard growth conditions were treated with 25, 50, and 
$100 \mathrm{mM} 2 \mathrm{DG}$ in $0.5 \mathrm{X}$ liquid MS medium in the dark for $3 \mathrm{~h}$ at $140 \mathrm{rpm}$.

\section{Abscisic Acid and Salt Treatments}

Five-days old uniformly grown Col-0 seedlings grown under standard growth condition were used for $\mathrm{ABA}$ as well as $\mathrm{NaCl}$ treatment. The seedlings were treated with $10 \mu \mathrm{M} \mathrm{ABA}$ in $0.5 \mathrm{X}$ liquid MS medium for $30 \mathrm{~min}, 3$ and $6 \mathrm{~h}$ time points at $22^{\circ} \mathrm{C}$ at $140 \mathrm{rpm}$ in the light. For salt treatment, the seedlings were subjected to $150 \mathrm{mM} \mathrm{NaCl}$ treatment in $0.5 \mathrm{X}$ liquid MS medium for $3 \mathrm{~h}$ at $22^{\circ} \mathrm{C}$ at $140 \mathrm{rpm}$ in the light.

\section{Gene Expression Analysis}

RNA isolation and cDNA preparation were performed as described previously (Mishra et al., 2009). qRT-PCR were done with 1:50 diluted cDNA samples with SYBR-Green PCR master mix in 384-well optical reaction plates employing Applied Biosystems 7500 Fast Real-Time PCR System (Applied Biosystems, USA). The primers were prepared from the transcript sequence of genes using PRIMER EXPRESS version v3.0 (Applied Biosystems, USA) with default parameters. 18S rRNA and UBQ10 were as used endogenous controls and relative quantification of the mRNA level of candidate genes were calculated by $\Delta \Delta \mathrm{CT}$ method (Livak and Schmittgen, 2001). Primers used for qRT-PCR experiments are given in Supplementary Table S1. The heat maps were generated from the gene expression data using MultiExperiment Viewer $(\mathrm{MeV}$, v4.8; Saeed et al., 2006). The hierarchical clustering of genes was performed by Pearson correlation algorithm with average linkage clustering.

The digital gene expression analysis under ABA and abiotic stress treatments was done using the AtGenExpress data available through Arabidopsis eFP Browser (Kilian et al., 2007; Winter et al., 2007; Goda et al., 2008). The expressions in the treatment time points were calculated using control expression values and heat map and hierarchical clustering were done as described above.

\section{Promoter: GUS Line Construction and Starvation Assay}

For promoter: GUS transcriptional fusion constructs (pFLZ1::GUSA, pFLZ6::GUSA, and pFLZ8::GUSA), primers designed from $2 \mathrm{~kb} 5^{\prime}$ UTR upstream region of FLZ1 and FLZ6 and $1.5 \mathrm{~kb} 5^{\prime}$ UTR upstream region of FLZ8. Primers used for cloning are listed in Supplementary Table S1. The amplified products were cloned in pMDC164 vector and transformed into Col-0 plants through floral dip transformation method (Clough and Bent, 1998; Curtis and Grossniklaus, 2003). The transformants were selected on 0.5X MS plates supplemented with $15 \mu \mathrm{g} / \mathrm{ml}$ hygromycin A and homozygous lines were identified in T3 generation. For starvation assay of promoter:: GUS lines, leaves of the same developmental stage from the rosette plants grown in standard growth conditions were detached and kept in petri plates with soaked filter paper in the dark for 1 and 2 days. The leaves were
GUS stained for $1 \mathrm{~h}$ as described previously (Jefferson et al., 1987).

\section{Results}

\section{Response of Arabidopsis thaliana FLZ Gene Family Genes Toward Cellular Energy Fluctuation}

The microarray-based expression analysis and protein-protein interaction analysis with KIN10/11 suggests the involvement of FLZ genes in low-energy stress (Nietzsche et al., 2014). In order to study the response of FLZ gene family genes in lowenergy stress at the transcriptional level, we did an extensive energy depletion and replenishment assay using 5-days old Col0 seedlings (Supplementary Figure S1A). The expression of all 18 A. thaliana FLZ genes was analyzed at different time points of low-energy stress and energy replenishment conditions (Figure 1A). It was found that the transcript levels of a group of genes were rapidly decreased on the onset of low-energy stress and these genes formed a distinct cluster in the heat map. The expression of these genes was significantly down-regulated in response to mild as well as prolonged energy depletion and their expressions were rapidly up-regulated in response to sugar replenishment (Supplementary Figure S1B). FLZ1, FLZ2, FLZ3, FLZ5, FLZ8, and FLZ14 belong to this cluster of genes whose levels are rapidly perturbed in response to energy depletion and replenishment. Among other members of this gene family, FLZ12, FLZ7, and FLZ15 do not show any significant difference in the expression in all treatments studied. FLZ4, FLZ10, FLZ11, and FLZ16 showed significant up-regulation of transcript level in the mild energy depletion. However, prolonged energy depletion significantly reduced their expression and sugar replenishment restored their expression to control level in most of these genes. FLZ9 showed a mild repression of transcript level at the onset of energy depletion; however, it showed significant repression of transcript levels after sugar replenishment. Interestingly, prolonged starvation significantly induced the transcript levels of FLZ6 and FLZ17/18 suggesting that these genes may be involved in the regulation of growth under prolonged low-energy stress. Consistent with this, it was found that the level of these two genes gradually reached the control level after energy is replenished to the plant. The levels were further reduced during prolonged incubation in energy-rich conditions (Figure 1A).

In order to validate the results obtained from energy depletion and replenishment assay, the expression of two selected FLZ genes was analyzed in samples treated with increasing concentrations of glycolysis inhibitor 2DG (Figure 1B). 2DG is a non-metabolizable glucose analog which is also a substrate of hexokinase and blocks the glycolytic pathway through competitive inhibition which ultimately leads to energy depletion in the cell (Wick et al., 1957). Increasing concentrations of 2DG significantly repressed the level of FLZ2 which was found to be negatively regulated during low-energy stress in the previous experiment. FLZ6, which was found to be positively regulated by prolonged low-energy stress, was found to be significantly up-regulated in response to $2 \mathrm{DG}$ treatment. These 
A
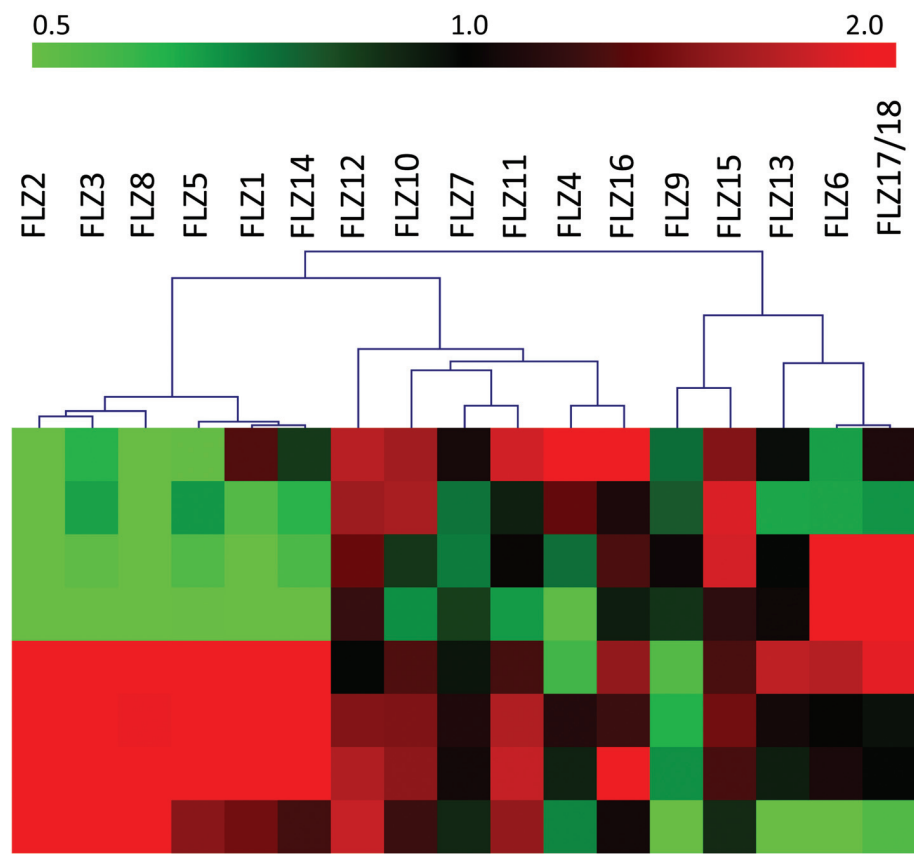

$\mathrm{S} 3 \mathrm{~h}$

$\mathrm{S} 6 \mathrm{~h}$

$\mathrm{S} 12 \mathrm{~h}$

$\mathrm{S} 24 \mathrm{~h}$

$\mathrm{R} 3 \mathrm{~h}$

$\mathrm{R} 6 \mathrm{~h}$

R $12 \mathrm{~h}$

R 24h

C

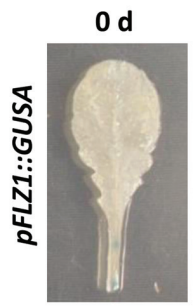

2 d

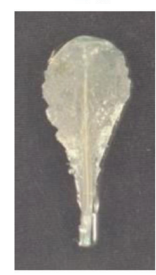

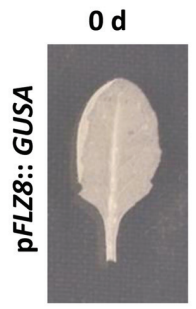

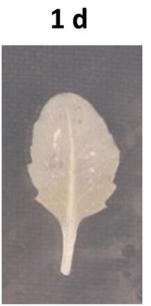

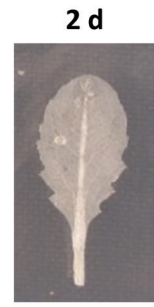

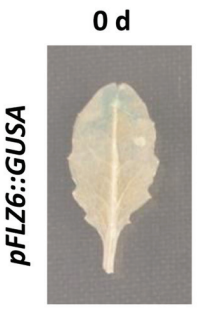

FLZ2
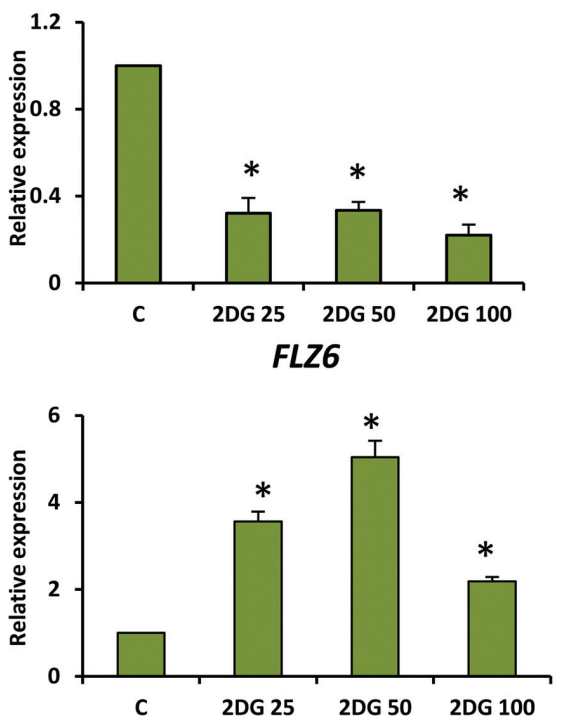

FIGURE 1 | Expression of Arabidopsis thaliana FLZ gene family during low-energy stress and replenishment. (A) Expression of $A$. thaliana $F L Z$ gene family under energy starvation and replenishment studied with QRT-PCR. S denotes 'starvation' and R denotes 'recovery.' The expression in 0.5X MS grown plants was taken as the control to compare the expression in low-energy levels and replenishment series. The genes were subjected to hierarchical clustering based on their response toward these treatments. UBQ10 was used as endogenous control. (B) Expression of FLZ2 and FLZ6 in response to increasing concentration of 2DG $(25,50$, and $100 \mathrm{mM})$. The bars represent the average of two independent biological experiments with three technical replicates each and error bars represent SE. UBQ10 was used as endogenous control. Asterisk indicates a significant difference of expression in the treatment compared to control $(P<0.005$, Student's $t$-test). (C) The response of $p F L Z 1:: G U S A, p F L Z 8:: G U S A$, and pFLZ6::GUSA transcriptional-reporter lines toward energy starvation for 0 , 1, and 2 days.

results validate the observations of the previous experiment. In order to further confirm these results, we used reporter lines of starvation-repressible and sugar-inducible genes FLZ1 and FLZ8 ( $p F L Z 1:: G U S A$ and $p F L Z 8:: G U S A$ ) and prolonged starvationinducible gene FLZ6 (pFLZ6::GUSA). Uniformly grown leaves of the same developmental stage were subjected to low-energy stress for 1 and 2 days and the GUS activity was compared with the leaves grown in normal conditions (Figure 1C). The GUS activity was found to be too low in the untreated leaves which is consistent with the earlier observation that the expression of these genes are too low in the mature leaves compared to other tissues (Jamsheer et al., 2015). The starvation could not induce the GUS activity in the leaves of $p F L Z 1:: G U S A$ and pFLZ8::GUSA lines. However; low-energy stress induced GUS expression in pFLZ6::GUSA line. All these results conclusively suggest that FLZ gene family contain genes which are positively and negatively regulated during low and high energy levels in the plant.

\section{Response of $\boldsymbol{F L Z}$ Genes Toward Sugars}

In the energy depletion and replenishment assay, it was found that the expression of many genes was positively or negatively regulated by sucrose replenishment. In order to dissect how the FLZ genes respond to sugar and energy rich condition in the cell, 24 h energy-starved Col-0 plants were treated with glucose, sucrose, mannose, and sugar alcohol mannitol. In order to rule out the effects of sugars other than the nutrient effect, seedlings were also treated with a non-metabolizable and nontoxic glucose analog, 3-O-methyl glucose (3-OMG; Cortès et al., 2003). Further, mannitol was used as an additional osmotic control to rule out the involvement of osmotic regulation of FLZ genes in the results. Among sugars, glucose, and sucrose profusely 
altered the transcript level of many genes (Supplementary Figure S2).

Based on their response toward glucose and/or sucrose treatment, the FLZ gene family was divided into three distinct classes. The transcript level of class I genes was induced by $\geq 10$ fold by glucose and/or sucrose (Figure 2A). FLZ1, FLZ2, FLZ5, FLZ8, and FLZ14 genes belong to this class of high sugar-inducible genes. Class II genes are medium sugar-inducible genes which are induced by $\geq 2$ fold by glucose and/or sucrose (Figure 2B). Class III includes sugar non-responsive genes as well as sugar-repressible genes (Figure 2C). FLZ6 could not display any significant response toward sugar treatments (Supplementary Figure S2). Interestingly, the levels FLZ7, FLZ9, FLZ12, and $F L Z 17 / 18$ were found to be significantly decreased in glucose, sucrose or in both sugars suggesting that these genes are sugarrepressed genes.

Mannose could also affect the transcription of many genes albeit to a lesser extent (Supplementary Figure S2). Among the sugar-induced genes, FLZ1, FLZ2, FLZ3, FLZ4 were found to be up-regulated in the presence of mannose. Similarly, sugar down-regulated genes such as FLZ7, FLLZ9, and FLZ17/18 were found to be also downregulated by mannose. FLZ12, which is specifically repressed by sucrose showed highest positive response toward mannose treatment.

\section{Role of Different Sugar Signaling Pathways in the Sugar Responsiveness of FLZ Genes}

It is already identified that different sugar signaling pathways act individually or along with other sugar signaling pathways to regulate the expression of sugar-responsive genes. The role of HXK1-dependent glucose signaling pathway is attributed to many glucose-mediated responses in plants (Mishra et al., 2009). Consistent with this, the glucose-induced up-regulation of key transcription factors involved in the aliphatic glucosinolate biosynthesis is completely abolished in the gin2-1 mutant (Miao et al., 2013). Similarly, the glucose-dependent repression of TANDEM ZINC FINGER 1 transcription is dependent on HXK1-signaling (Lin et al., 2011). These reports suggest that downstream effects of glucose at the transcriptional level are partly mediated by HXK1-dependent glucose signaling pathway. Similarly, the role of TOR and SnRK1 dependent energy signaling pathway is also implicated in the regulation of many sugar responsive genes (Baena-González et al., 2007; Ramon et al., 2008; Xiong et al., 2013).

Genetic and pharmacological approaches were used to identify the role of different sugar signaling pathways on the sugar responsiveness of FLZ genes. To study whether HXK1-dependent glucose signaling has any role in the transcriptional up-regulation of FLZ genes, we checked the glucose responsiveness of sugarinducible FLZ genes in the gin2-1 mutant. It was found that the transcript induction of $F L Z$ genes after glucose treatment was significantly reduced in the gin2-1 mutant (Figure 3). Abolition of the HXK1-dependent glucose signaling pathway could only partially affect the sugar-dependent activation of FLZ genes suggesting the involvement of other sugar signaling pathways in this response. The treatment of $2 \mathrm{DG}$ significantly reduced the transcript level of FLZ2 which suggest that ultimately cellular energy level regulates the expression of sugar-induced FLZ genes. To confirm this observation, sugar depleted seedlings were treated with sugars alone or in combination with chemicals which inhibit glycolysis and various steps of oxidative phosphorylation. The glycolysis inhibitor 2DG, the mitochondrial electron transport blocker AMA and mitochondrial uncoupling agents CCCP and DNP were used for studying the effect of metabolic signaling on the induction of FLZ genes (Xiong et al., 2013). It was found that the induction of transcript level by sugars was severely abolished when sugars were given in combination with metabolic inhibitors (Figure 4).

FiguRE 2 I Sugar sensitivity of Arabidopsis thaliana FLZ gene family. The A. thaliana FLZ gene family was classified into three groups based on their
response toward sugar treatments in qRT-PCR experiments. The expression in 0\% sugar grown plants was taken as the control for comparing the expression in
other treatments and 18S rRNA was used as endogenous control. (A) High sugar-inducible genes (Class I, induced by $\geq 10$ fold by glucose and/or sucrose).
(B) Medium sugar-inducible genes (Class II, induced by $\geq 2$ fold by glucose and/or sucrose). (C) Sugar non-responsive and sugar-repressible genes (Class III,
significantly repressed by glucose and/or sucrose).




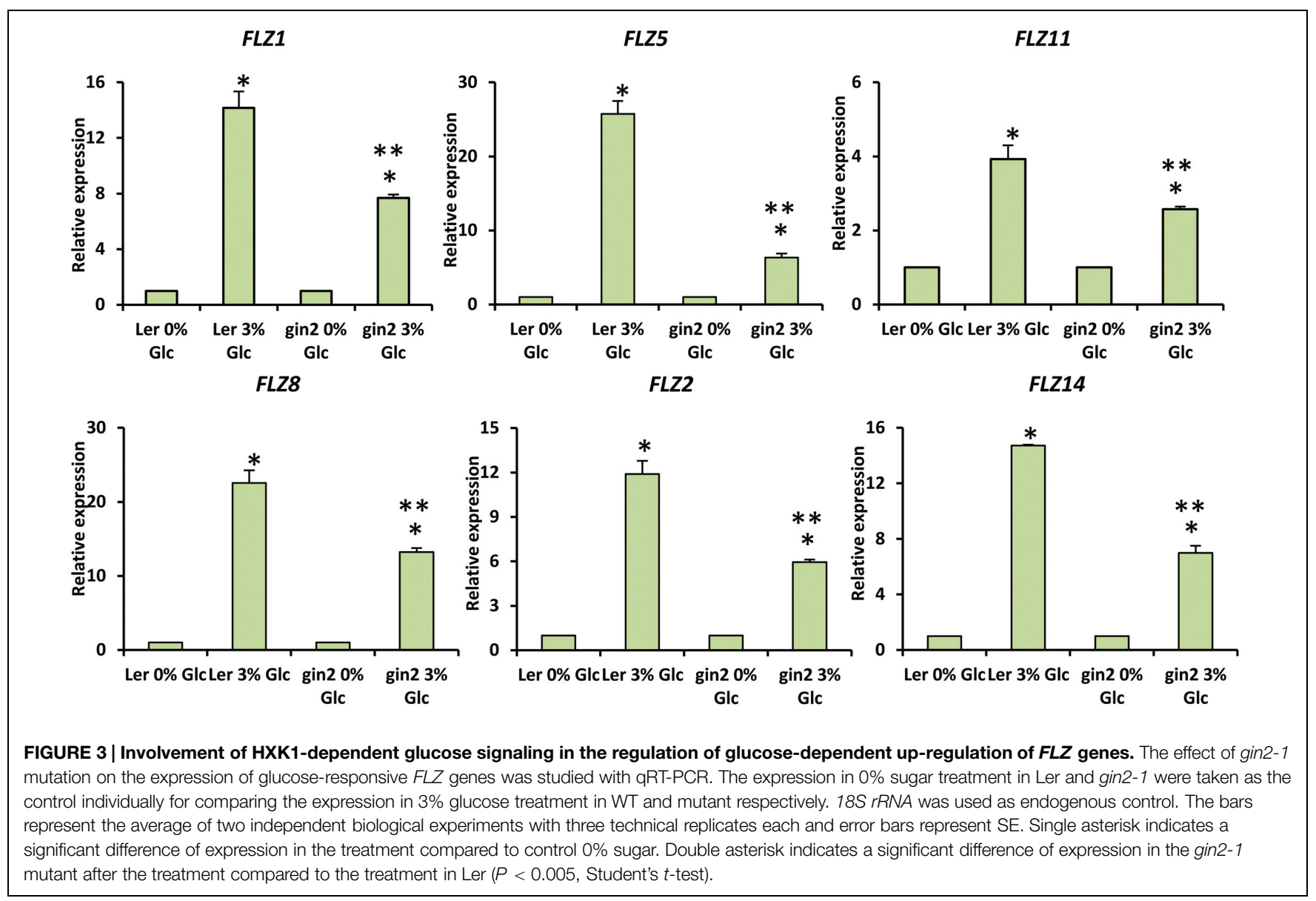

\section{Response of $F L Z$ Genes towards Different Abiotic Stresses and ABA Treatment}

Over-expression of a wheat FLZ gene TaSRHP in A. thaliana resulted in enhanced salt stress tolerance. TaSRHP found to be a positive regulator of many stress-related genes (Hou et al., 2013). This result suggests an involvement of FLZ genes in salt stress response. Most of the FLZ genes were found to be responsive to the cellular energy deficit which is also caused by stresses. We made use of available microarray data to investigate whether salt stress directly regulates the expression of FLZ genes and identified that salt stress differentially regulate the expression of all FLZ genes (Figure 5A). Genes like FLZ2, FLZ10, FLZ11, and FLZ15 were found to be constantly up-regulated during salt stress. However, most of the genes showed temporal response toward the salt treatment. In order to validate the microarray data, 5-days old seedlings were subjected to salt stress for $3 \mathrm{~h}$ and the expression level of 4 FLZ genes which were shown differential regulation in the microarray data was analyzed (Figure $5 \mathbf{B}$ ). As observed in the microarray data, FLZ10 and FLZ11 were found to be moderately up-regulated in response to $3 \mathrm{~h}$ salt stress. Similarly, moderate down-regulation of FLZ13 was observed in the qRT-PCR data also. FLZ17/18, which were found to be profusely up-regulated in almost all stages in the microarray data was found to be profusely up-regulated in the salt treated 5-days old seedlings too.
We also analyzed the expression pattern of FLZ genes under other abiotic stress using available microarray data and it was found that the expression of these genes were considerably fluctuated in response to different abiotic stresses (Supplementary Figure S3). In general, it can be seen that the response of these genes toward different stresses are more or less spatiotemporal. The varied response of FLZ genes toward different abiotic stress prompted us to investigate the response of these genes toward ABA. Digital expression analysis identified that many members are of this gene family are differentially regulated in response to ABA treatment (Figure 5C). Most of the genes were found to be positively regulated by ABA while few genes like FLZ13 were found to be down-regulated. In order to validate the microarray data, we analyzed the response of two FLZ genes in 5-days old seedlings treated with ABA at three different time points (Figure 5D). Among these two genes, the ABAresponsiveness of FLZ9 is already reported earlier which can be used as excellent marker for validating microarray data (He and Gan, 2004). As observed in the microarray data, the expression of FLZ9 was found to be gradually increased in response to ABA treatment. Similarly, the expression of FLZ17/18 was also found to be significantly increased in response to $3 \mathrm{~h}$ ABA treatment. Taken together, all these results suggest the possible involvement on FLZ genes in plant growth under stress. 
A
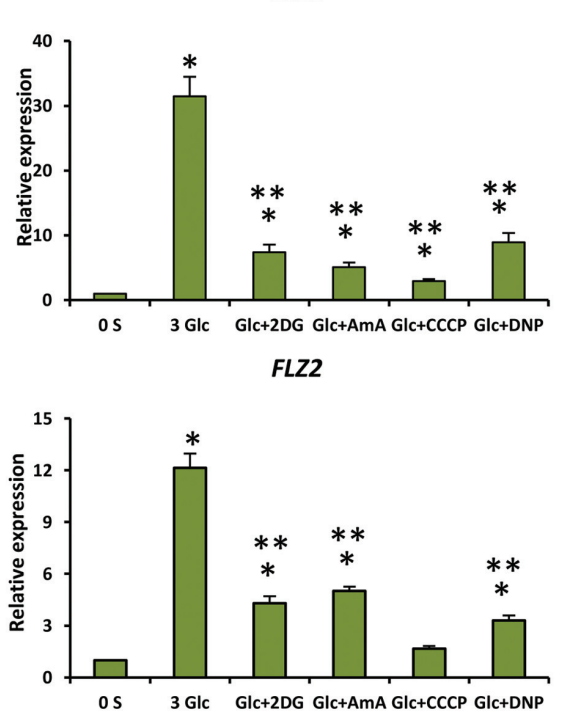

FLZ8

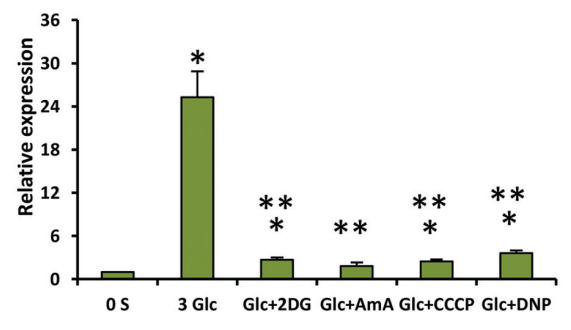

FLZ10

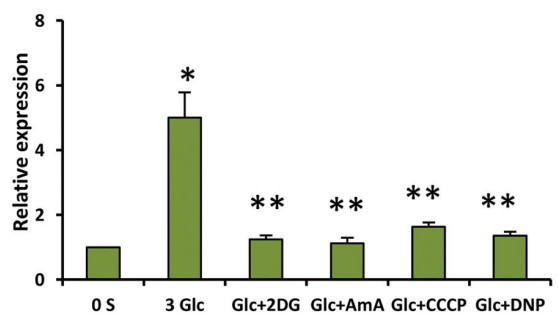

B
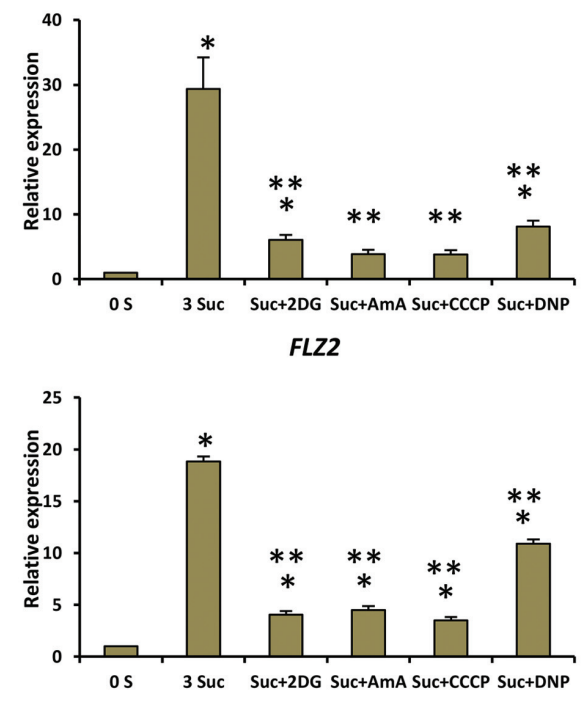

FLZ8

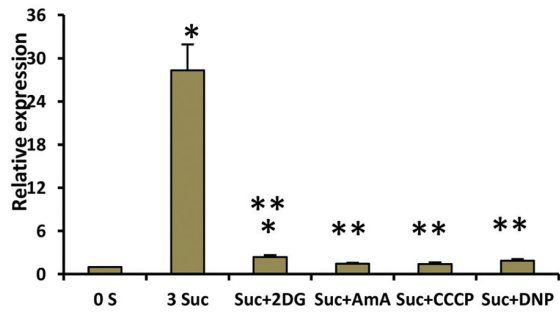

FLZ10

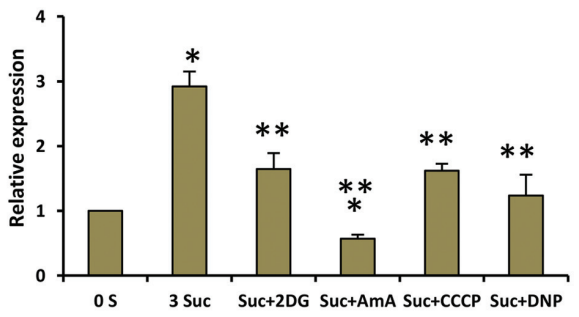

FIGURE 4 | Involvement of energy signaling in the regulation sugar-dependent up-regulation of $\boldsymbol{F L} \boldsymbol{Z}$ genes. The effect of metabolic inhibitors on the sugar-mediated transcriptional up-regulation of $F L Z$ genes was studied by qRT-PCR. (A) The effect of metabolic inhibitors on the glucose-sensitivity of $F L Z$ genes. (B) The effect of metabolic inhibitors on the sucrose-sensitivity of $F L Z$ genes. The expression in $0 \%$ sugar grown plants was taken as the control for comparing the expression in 3\% sugar treatment alone or with metabolic inhibitors. UBQ10 was used as endogenous control. The bars represent the average of two independent biological experiments with three technical replicates each and error bars represent SE. Single asterisk indicates a significant difference of expression in the treatment compared to control 0\% sugar. Double asterisk indicates a significant difference of expression in the treatment compared to 3\% Glc or 3\% Suc $(P<0.005$, Student's $t$-test).

\section{Involvement of SnRK1 in the Regulation of FLZ Genes}

It is already established that SnRK1 regulates the expression of genes involved in the mitigation of multiple stresses including low-energy stress (Baena-González et al., 2007). The overexpression of SnRK1.1 in A. thaliana resulted in enhanced starvation tolerance, late senescence, and perturbation in the normal developmental processes (Baena-González et al., 2007). In order to investigate whether the FLZ genes have any connection with the conserved SnRK1 signaling cascade, we checked the expression of selected FLZ genes from all three classes in KIN10 over-expression line KIN10 OE2 (Supplementary Figure S4). It was found that SnRK1 repress the transcription of sugar-inducible FLZ genes (Figure 6A). The degree of repression in high sugar-inducible genes (Class I) like FLZ2, FLZ3, and FLZ8 were found to be more severe compared to the repression in medium sugar-inducible gene (Class II) FLZ11. The expression of sugar-repressible genes (Class III) FLZ9 and 


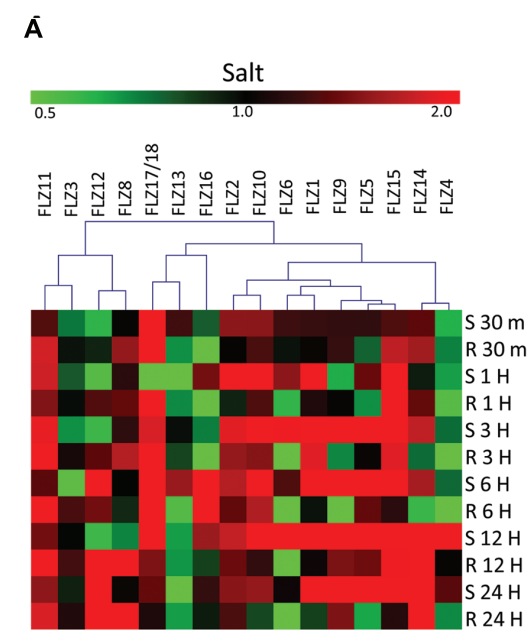

C
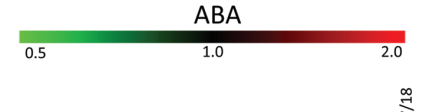

B
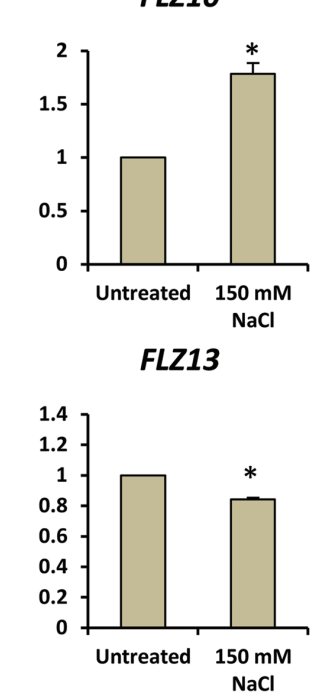

FLZ9
FLZ11
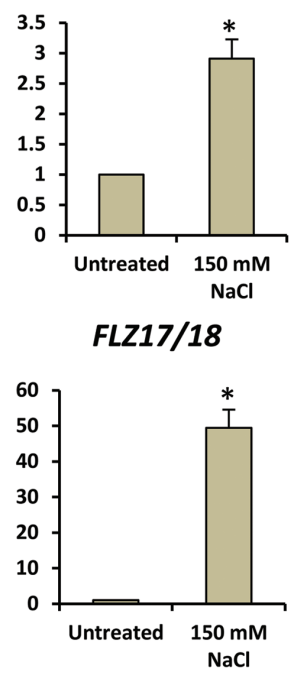

D
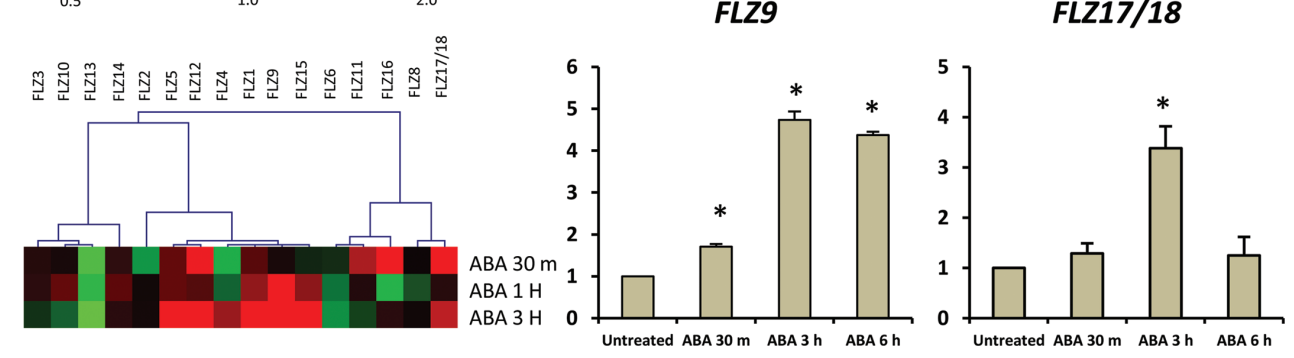

FIGURE 5 | Expression of $F L Z$ genes during salt stress and abscisic acid. The impact of salt stress and ABA on the expression of $F L Z$ genes was studied using available microarray data and by qRT-PCR. (A) The expression of $F L Z$ genes during salt stress from available microarray data. The expression data was downloaded from the AtGenExpress global stress expression data set (Kilian et al., 2007) available through Arabidopsis eFP Browser (Winter et al., 2007). The genes were subjected to hierarchical clustering based on their response toward salt stress. S denotes 'shoot' and R denotes 'root.' (B) The expression of selected FLZ genes in 5-days old seedlings under salt stress. (C) The response of FLZ genes toward ABA from available microarray data available through Arabidopsis eFP Browser (Winter et al., 2007; Goda et al., 2008). (D) The expression of two selected FLZ genes in 5-days old seedlings treated with ABA at three different time points. In both salt stress and ABA treatments, the expression in 5-days old Col seedlings grown on 0.5X MS was used as the control to compare the transcript level of FLZ genes under salt stress and ABA. 18S rRNA was used as endogenous control. The bars represent the average of two independent biological experiments with three technical replicates each and error bars represent SE. Asterisk indicates a significant difference of expression in the treatment compared to control $(P<0.005$, Student's $t$-test).

FLZ17/18 were found to be significantly increased in KIN10 overexpression line (Figure 6B). However, the expression of sucroserepressible and mannose-inducible gene FLZ12 was found to be unperturbed. Mannose-repressible FLZ13 level was also found to be unchanged in the KIN10 over-expression lines suggesting that the regulation of SnRK1 on FLZ gene is directly dependent on their response toward metabolizable sugars such as glucose and sucrose.

\section{Discussion}

\section{FLZ Genes are Transcriptionally Regulated by Sugars, ABA, and Abiotic Stresses}

It is already reported that FLZ9 expression is induced during leaf senescence and nutrient deficiency differentially regulates FLZ genes (He et al., 2001; Nietzsche et al., 2014). In our analysis, it was found that the expression of most of the FLZ gene family members is differentially regulated in response to sugar treatment and under energy depleted and replenished conditions. The expressions of these genes were found to be highly regulated by glucose, sucrose and to a very less extent by mannose which confirms that this response is dependent on sugar signaling and metabolism but not dependent on the osmotic effects of sugars. We could classify the FLZ genes into three classes based on their response toward sugars. Each class contains genes in which some of them is phylogenetically closer and some of them are phylogenetically very distant suggesting that the specific response toward sugars is not confined to any specific clad defined in an earlier study (Jamsheer and Laxmi, 2014). The expression of all high sugar-inducible genes (Class I) was repressed in response to low-energy stress and their levels were rapidly increased during 


\section{A}

FLZ2

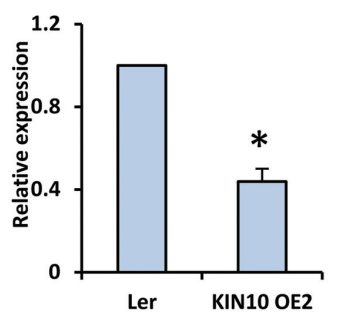

B

FLZ9

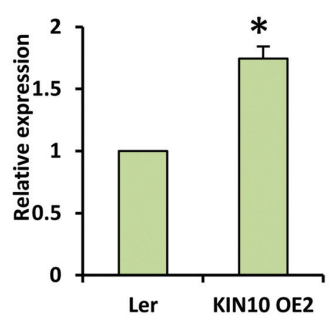

FLZ3

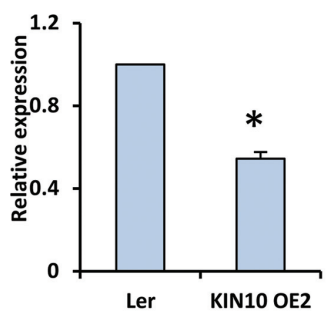

FLZ12

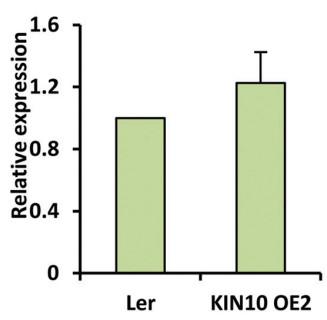

FLZ8

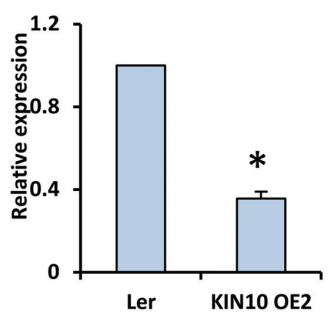

FLZ13

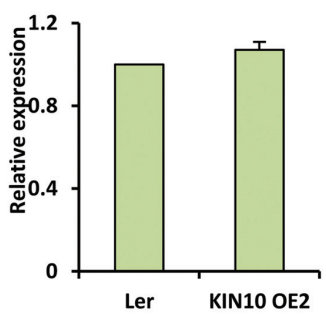

FLZ11
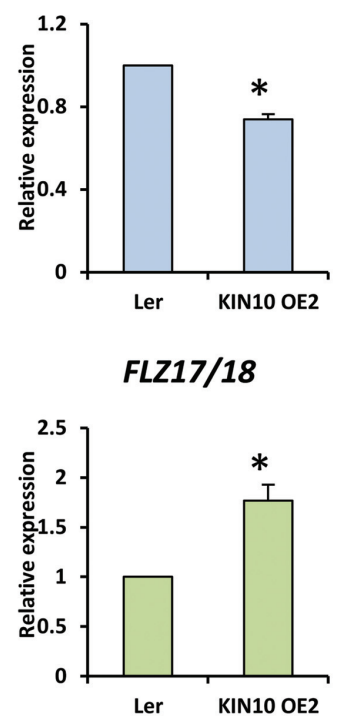

FIGURE 6 | Expression of $F L Z$ genes in SnRK1.1 over-expression line. The transcript level of selected FLZ genes in KIN10 OE2 seedlings were studied with qRT-PCR. (A) The expression of sugar-inducible (Class I and II) FLZ genes in KIN10 OE2. (B) The expression of Class III FLZ genes in KIN10 OE2. The expression in 5-days old Ler was used as the control to compare the transcript level of FLZ genes in KIN1O OE2. UBQ10 was used as endogenous control. The bars represent the average of two independent biological experiments with three technical replicates each and error bars represent SE. Single asterisk indicates a significant difference of expression in KIN10 OE2 compared to Ler $(P<0.005$, Student's $t$-test).

sugar replenishment. Similarly, FLZ3 which is a paralog of high sugar-inducible genes FLZ1 and FLZ2 showed a similar response. In medium sugar-inducible group (Class II), many genes found to be were up-regulated in response to mild low-energy stress while prolonged stress repressed their levels. Some of the sugarrepressible genes (Class III) identified from the sugar sensitivity assay showed induction of transcript levels in response to lowenergy stress. FLZ6 and FLZ17/18 were found to be responsive to prolonged starvation. These results suggest that majority of FLZ gene family members are responsive to sugar levels and cellular starvation. These results were further supported by GUS assay and $2 \mathrm{DG}$ treatment.

It is already well known that sugars transcriptionally regulate a large number of genes which belongs to diverse cellular processes. These sugar-regulated genes include genes involved in primary and secondary metabolism, hormone signaling and developmental processes such as phase transition, flowering, senescence etc. (Price et al., 2004; Li et al., 2006; Mishra et al., 2009; Xiong et al., 2013). Functional analysis of these genes deciphered molecular mechanisms by which sugars regulate different aspects of plant development. For example, the sugarinduced expression of nucelolin-1 in A. thaliana is important for ribosome synthesis (Kojima et al., 2007). Similarly, the glucoseTARGET OF RAPAMYCIN (TOR) signaling cascade regulates the transcription of many genes including cell cycle genes which is important in the establishment of early seedling growth (Xiong et al., 2013). From our analysis, it has been found that the cellular sugar and energy level is an important regulator of transcription of many FLZ genes. Similar functional analysis of individual FLZ genes will give more information on the molecular aspects of the sugar-mediated regulation of plant growth. Interestingly, in an earlier study, we have identified that most of the FLZ genes are differentially regulated during vegetative-to-reproductive phase transition (Jamsheer et al., 2015). The role of sugars on the developmental phase transition and flowering is already known. Sugars known to regulate these processes by controlling the activity of microRNAs and other well know regulators involved in the developmental phase transition and flowering (Seo et al., 2010; Wahl et al., 2013; Yang et al., 2013; Yu et al., 2013). The differential regulation of FLZ genes during vegetative-toreproductive phase transition further supports the possible role of FLZ gene family in the sugar-mediated regulation of plant growth. The interaction of these proteins with kinase subunits of SnRK1 and regulatory subunit of TOR kinase also suggest their involvement of FLZ genes in the sugar-mediated regulation of plant growth (Arabidopsis Interactome Mapping Consortium, 2011; Nietzsche et al., 2014). Our transcriptional studies and earlier protein: protein interaction studies could suggest a link between sugars and FLZ gene family; however, more molecular studies are needed to establish this connection.

Transcriptional studies also identified that different abiotic stresses and ABA can regulate the expression of many FLZ genes. These results suggest the possible involvement of FLZ genes in mediating adaptive growth under abiotic stress conditions. There are already few reports which link FLZ genes in providing stress tolerance and involvement in ABA-directed processes. Over-expression of a wheat FLZ gene, TaSRHP in A. thaliana positively regulated the expression of many stress-related genes 
and enhanced resistance toward different abiotic stresses (Hou et al., 2013). The FLZ9/MARD1 gene of A. thaliana was found to involved in ABA-mediated seed dormancy ( $\mathrm{He}$ and Gan, 2004). Similarly, the interplay between sugars and stress signaling is well established. A large overlap between sugar- and stress-responsive genes is already reported by different groups suggesting the role of sugars and cellular energy in providing stress-tolerance (Price et al., 2004; Li et al., 2006). Disruption of many genes resulted in perturbation of both ABA- and sugarmediated responses further suggest an overlap between glucose and stress signaling (Arenas-Huertero et al., 2000; Brocard et al., 2002). Our transcriptional studies firmly established that the $F L Z$ genes are regulated by sugars, ABA, and abiotic stresses. Since sugar and cellular energy status is important in dealing stresses, this class of genes which are co-regulated by both sugars and stresses might be important in the regulation of adaptive responses toward stress and stress-derived energy depletion (Figure 7). The studies in this direction may open a novel molecular pathway by which sugars regulate adaptive growth during stress.

\section{The Sugar-Induced Expression of $F L Z$ Genes is Dependent on HXK1-Dependent Signaling and Metabolism-Dependent Energy Signaling}

Different glucose signaling pathways are implicated in the control of sugar-regulated gene expression in both distinct and overlapping manner (Miao et al., 2013; Xiong et al., 2013). In our study, we found that the sugar-induced expression of FLZ genes is regulated by two different sugar signaling pathways. Abolition of HXK1-dependent pathway reduced the expression of FLZ genes considerably after sugar treatment suggesting that HXK1-dependent signaling acts as a positive regulator of glucoseinduced expression of FLZ genes. This pathway is independent of the kinase activity of HXK1 and decoupled from the sugar metabolism-dependent signaling (Moore et al., 2003). HXK1 form complex with $\mathrm{H}^{+}$-ATPase $\mathrm{B} 1$ and the $19 \mathrm{~S}$ regulatory particle of proteasome subunit in the nucleus and sugar-regulated expression of many genes are reported to be dependent on this pathway (Cho et al., 2006; Lin et al., 2011; Miao et al., 2013). However, in case of sugar-induced FLZ genes, the abolition of the HXK1-dependent pathway could only partially hamper the transcript induction suggesting the involvement of other sugar signaling pathways.

The expression of FLZ genes was severely abolished when the cellular respiratory pathway was blocked independently at glycolysis and oxidative phosphorylation stages implying the role of metabolism-dependent energy signaling as the pivotal regulator of the sugar-induced FLZ gene expression. This observation is further supported by the contrasting response of FLZ2 and FLZ6 toward 2DG treatment. The metabolism dependent energy signaling pathway is implicated in controlling TOR and SnRK1 signaling (Nunes et al., 2013; Xiong et al., 2013). It is earlier reported that glucose-control of cell division, meristem activation, and the developmental transition is dependent on metabolism-dependent energy signaling pathway and independent of HXK1-dependent glucose signaling pathway. Understandably, all major hormone signaling pathways and the

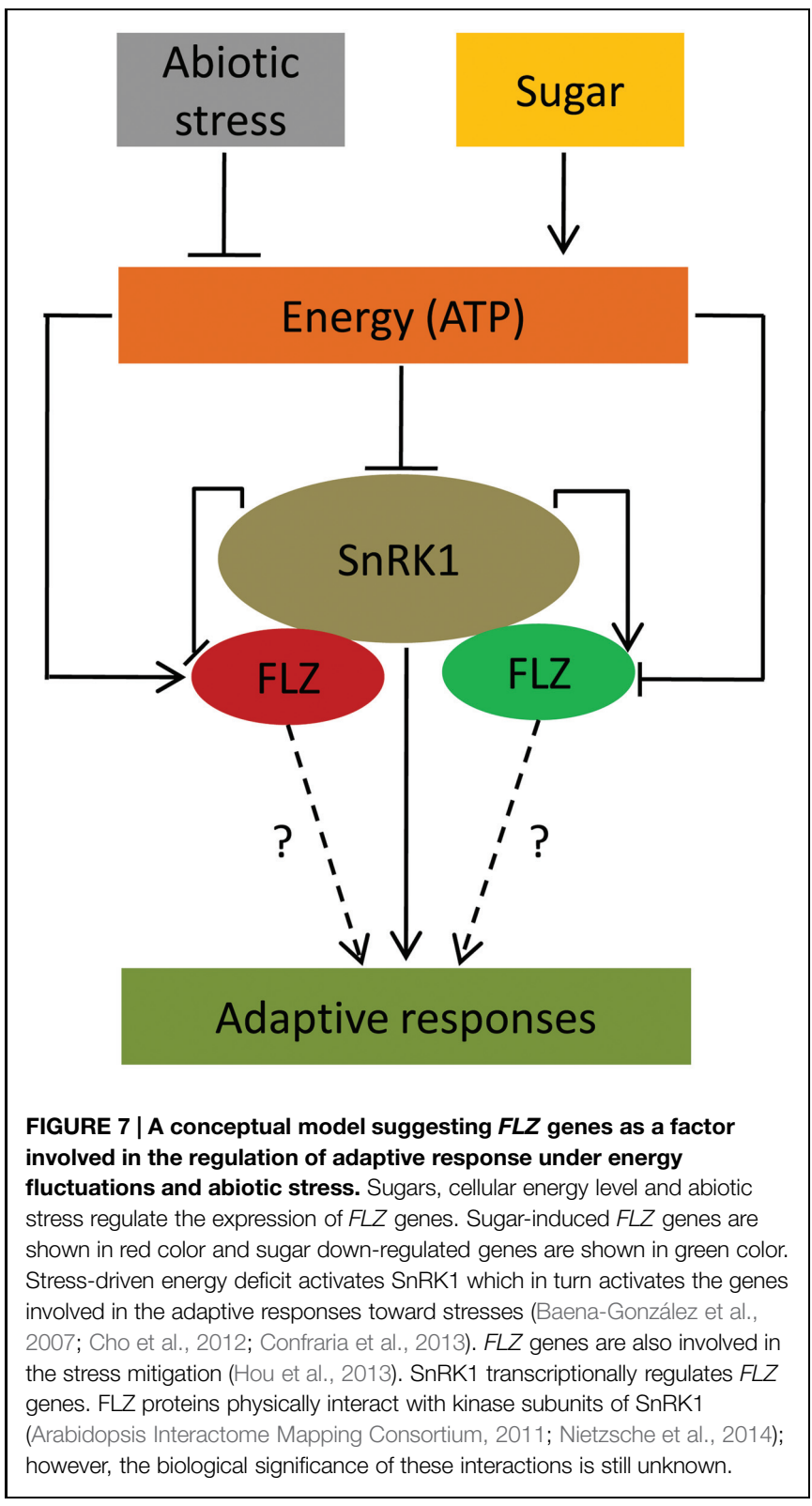

HXK1-dependent glucose signaling found to be downstream to the metabolism-dependent control of plant growth (Xiong et al., 2013). Our results suggest that induction of FLZ genes under sugar and energy rich conditions is controlled by metabolism-dependent energy signaling. The HXK1-dependent glucose signaling acts as a positive regulator of glucoseinduced transcriptional up-regulation of FLZ genes. This regulation may be important for the fine tuning of FLZ gene function in response to sugar and energy signaling. Besides, the dual regulation of sugar-dependent transcriptional induction of FLZ genes by both HXK1-dependent glucose signaling and metabolism-dependent energy signaling suggest the possibility that FLZ genes might be acting as a crosstalking hub for different sugar signaling pathways established in plants. 


\section{SnRK1 Regulation of $F L Z$ Gene Transcription}

The gene expression analysis in KIN10 over-expression identified that SnRK1 regulates the transcription of FLZ genes. Interestingly, this regulation of SnRK1 on the transcription of $F L Z$ genes found to have a connection with the sugar-response of these genes. The sugar-inducible FLZ genes were found to be repressed while the sugar-repressible genes were found to up-regulated in the KIN10 over-expression line. The repression was found to be more severe in high sugar-inducible genes (Class I) compared to medium sugar-inducible gene FLZ11 (Class II). Consistent with this hypothesis, it was found that KIN10 positively regulates the expression of class III sugarrepressible genes FLZ9 and FLZ17/18. These results provide a framework for the further studies regarding the role of FLZ gene in the regulation of stress responses, particularly during low-energy stress. This result suggests that SnRK1 is possibly working upstream to regulate the transcription of FLZ genes. It is already known that SnRK1 undertake a massive reprogramming of transcription during low-energy stress (Baena-González et al., 2007). Interestingly, the contrasting regulation of Class I and II genes and Class III genes in the KIN10 over-expression line add more complexity to the situation. Molecular study of this sugar-response dependent regulation of FLZ genes by SnRK1 would reveal the further intricacies of adaptive growth under energy stress. The genetic studies using FLZ gene mutants and over-expression studies can tell whether these genes work downstream to SnRK1 in this pathway. Besides, it is known that both kinase subunits of SnRK1 physically interact with all FLZ proteins in A. thaliana (Arabidopsis Interactome Mapping Consortium, 2011; Nietzsche et al., 2014). Identification of biological significance of these interactions will be crucial in the elucidation of the molecular regulation of energy signalingSnRK1 interaction. The FLZ proteins can be a downstream factor by acting as kinase substrate of SnRK1 or it is possible that this

\section{References}

Arabidopsis Interactome Mapping Consortium. (2011). Evidence for network evolution in an Arabidopsis interactome map. Science 333, 601-607. doi: $10.1126 /$ science. 1203877

Arenas-Huertero, F., Arroyo, A., Zhou, L., Sheen, J., and León, P. (2000). Analysis of Arabidopsis glucose insensitive mutants, gin5 and gin6, reveals a central role of the plant hormone ABA in the regulation of plant vegetative development by sugar. Genes Dev. 2000, 2085-2096. doi: 10.1101/gad.14.16. 2085

Baena-González, E., Rolland, F., Thevelein, J., and Sheen, J. (2007). A central integrator of transcription networks in plant stress and energy signalling. Nature 448, 938-942. doi: 10.1038/nature06069

Baena-González, E., and Sheen, J. (2008). Convergent energy and stress signaling. Trends Plant Sci. 13, 474-482. doi: 10.1016/j.tplants.2008.6.006

Brocard, I., Lynch, T. J., and Finkelstein, R. R. (2002). Regulation and role of the Arabidopsis abscisic acid-insensitive 5 gene in abscisic acid, sugar, and stress response. Plant Physiol. 129, 1533-1543. doi: 10.1104/pp.005793

Chen, X., Zhang, Z., Visser, R., Broekgaarden, C., and Vosman, B. (2013). Overexpression of IRM1 enhances resistance to aphids in Arabidopsis thaliana. PLoS ONE 8:e70914. doi: 10.1371/journal.pone.0070914

Cho, Y., Hong, J., Kim, E., and Yoo, S. (2012). Regulatory functions of SnRK1 in stress-responsive gene expression and in plant growth and development. Plant Physiol. 158, 1955-1964. doi: 10.1104/pp.111.189829 interaction regulate the kinase activity of SnRK1. Considering the regulation of sugar and energy level on SnRK1 activity and FLZ gene expression, both hypotheses deserve the merit for further studies. Low light and various abiotic stresses ultimately lead to energy deficit in the cell and the response of FLZ gene toward energy fluctuation and stress suggest that they are possibly involved in the regulation of adaptive responses which enable the plant to survive in these non-favorable conditions. More genetic, molecular and physiological studies are needed to decipher this pathway.

\section{Author Contributions}

Conceived and designed the experiments: MJK and AL. Performed the experiments: MJK. Analyzed the data: MJK and AL. Contributed reagents/materials/analysis tools: MJK and AL. Wrote the paper: MJK and AL.

\section{Acknowledgments}

We are grateful to the National Institute of Plant Genome Research Central Instrument facility (Real Time PCR division) for their assistance. This work was financially supported by the National Institute of Plant Genome Research (NIPGR) core grant and University Grants Commission, Government of India (research fellowship to MJK). Authors thank Prof. Filip Rolland for providing seeds of KIN10 OE2.

\section{Supplementary Material}

The Supplementary Material for this article can be found online at: http://journal.frontiersin.org/article/10.3389/fpls.2015.00746

Cho, Y., Yoo, S., and Sheen, J. (2006). Regulatory functions of nuclear Hexokinase1 complex in glucose signaling. Cell 127, 579-589. doi: 10.1016/j.cell.2006.09.028

Clough, S., and Bent, A. (1998). Floral dip: a simplified method for Agrobacteriummediated transformation of Arabidopsis thaliana. Plant J. 16, 735-743. doi: 10.1046/j.1365-313x.1998.00343.x

Colaneri, A., Tunc-Ozdemir, M., Huang, J., and Jones, A. (2014). Growth attenuation under saline stress is mediated by the heterotrimeric $\mathrm{G}$ protein complex. BMC Plant Biol. 14:129. doi: 10.1186/1471-2229-14-129

Confraria, A., Martinho, C., Elias, A., Rubio-Somoza, I., and Baena-González, E. (2013). miRNAs mediate SnRK1-dependent energy signaling in Arabidopsis. Front. Plant Sci. 4:197. doi: 10.3389/fpls.2013.00197

Cortès, S., Gromova, M., Evrard, A., Roby, C., Heyraud, A., Rolin, D. B., et al. (2003). In plants, 3-o-methylglucose is phosphorylated by hexokinase but not perceived as a sugar. Plant Physiol. 131, 824-837.

Curtis, M., and Grossniklaus, U. (2003). A gateway cloning vector set for highthroughput functional analysis of genes in planta. Plant Physiol. 133, 462-469. doi: 10.1104/pp.103.027979

Goda, H., Sasaki, E., Akiyama, K., Maruyama-Nakashita, A., Nakabayashi, K., Li, W., et al. (2008). The AtGenExpress hormone- and chemical-treatment data set: experimental design, data evaluation, model data analysis, and data access. Plant J. 55, 526-542. doi: 10.1111/j.0960-7412.2008.03510.x

He, Y., and Gan, S. (2004). A novel zinc-finger protein with a proline-rich domain mediates ABA-regulated seed dormancy in Arabidopsis. Plant Mol. Biol. 54, 1-9. doi: 10.1023/b:plan.0000028730.10834.e3 
He, Y., Tang, W., Swain, J. D., Green, A. L., Jack, T. P., and Gan, S. (2001). Networking senescence-regulating pathways by using Arabidopsis enhancer trap lines. Plant Physiol. 126, 707-716. doi: 10.1104/pp.126.2.707

Heilbronn, L., and Ravussin, E. (2005). Calorie restriction extends life span-but which calories? PLoS Med. 2:e231. doi: 10.1371/journal.pmed.0020231

Hey, S., Mayerhofer, H., Halford, N., and Dickinson, J. (2007). DNA sequences from Arabidopsis, which encode protein kinases and function as upstream regulators of Snf1 in yeast. J. Biol. Chem. 282, 10472-10479. doi: $10.1074 /$ jbc.m611244200

Hou, X., Liang, Y., He, X., Shen, Y., and Huang, Z. (2013). A novel ABA-responsive TaSRHP gene from wheat contributes to enhanced resistance to salt stress in Arabidopsis thaliana. Plant Mol. Biol. Rep. 31, 791-801. doi: 10.1007/s11105012-0549-9

Jamsheer, K. M., and Laxmi, A. (2014). DUF581 is plant specific FCS-Like Zinc Finger involved in protein-protein interaction. PLOS ONE 9:e99074. doi: 10.1371/journal.pone.0099074

Jamsheer, K. M., Mannully, C. T., Gopan, N., and Laxmi, A. (2015). Comprehensive evolutionary and expression analysis of FCS-Like Zinc finger gene family yields insights into their origin, expansion and divergence. PLOS ONE 10:e0134328. doi: 10.1371/journal.pone.0134328

Jefferson, R. A., Kavanagh, T. A., and Bevan, M. W. (1987). GUS fusions: betaglucuronidase as a sensitive and versatile gene fusion marker in higher plants. EMBO J. 6, 3901-3907.

Kilian, J., Whitehead, D., Horak, J., Wanke, D., Weinl, S., Batistic, O., et al. (2007). The AtGenExpress global stress expression data set: protocols, evaluation and model data analysis of UV-B light, drought and cold stress responses. Plant J. 50, 347-363. doi: 10.1111/j.1365-313x.2007.03052.x

Kojima, H., Suzuki, T., Kato, T., Enomoto, K., Sato, S., Kato, T., et al. (2007). Sugarinducible expression of the nucleolin-1 gene of Arabidopsis thaliana and its role in ribosome synthesis, growth and development. Plant J. 49, 1053-1063. doi: $10.1111 / j .1365-313 x .2006 .03016 . x$

Leon, P., and Sheen, J. (2003). Sugar and hormone connections. Trends Plant Sci. 8, 110-116. doi: 10.1016/s1360-1385(03)00011-6

Li, Y., Lee, K. K., Walsh, S., Smith, C., Hadingham, S., Sorefan, K., et al. (2006). Establishing glucose- and ABA-regulated transcription networks in Arabidopsis by microarray analysis and promoter classification using a relevance vector machine. Genome Res. 16, 414-427. doi: 10.1101/gr.4237406

Lin, P., Pomeranz, M., Jikumaru, Y., Kang, S., Hah, C., Fujioka, S., et al. (2011). The Arabidopsis tandem zinc finger protein AtTZF1 affects ABA- and GAmediated growth, stress and gene expression responses. Plant J. 65, 253-268. doi: $10.1111 / j .1365-313 x .2010 .04419 . x$

Livak, K., and Schmittgen, T. (2001). Analysis of relative gene expression data using real-time quantitative PCR and the 2(-Delta Delta C(T)) method. Methods 25, 402-408. doi: 10.1006/meth.2001.1262

Miao, H., Wei, J., Zhao, Y., Yan, H., Sun, B., Huang, J., et al. (2013). Glucose signalling positively regulates aliphatic glucosinolate biosynthesis. J. Exp. Bot. 64, 1097-1109. doi: 10.1093/jxb/ers399

Minina, E., Sanchez-Vera, V., Moschou, P., Suarez, M., Sundberg, E., Weih, M., et al. (2013). Autophagy mediates caloric restriction-induced lifespan extension in Arabidopsis. Aging Cell 12, 327-329. doi: 10.1111/acel.12048

Mishra, B., Singh, M., Aggrawal, P., and Laxmi, A. (2009). Glucose and auxin signaling interaction in controlling Arabidopsis thaliana seedlings root growth and development. PLoS ONE 4:e4502. doi: 10.1371/journal.pone.0004502

Moore, B., Zhou, L., Rolland, F., Hall, Q., Cheng, W. H., Liu, Y. X., et al. (2003). Role of the Arabidopsis glucose sensor HXK1 in nutrient, light, and hormonal signaling. Science 300, 332-336. doi: 10.1126/science.1080585

Nietzsche, M., Schießl, I., and Börnke, F. (2014). The complex becomes more complex: protein-protein interactions of SnRK1 with DUF581 family proteins provide a framework for cell- and stimulus type-specific SnRK1 signaling in plants. Front. Plant Sci. 5:54. doi: 10.3389/fpls.2014.00054

Nunes, C., O’Hara, L., Primavesi, L., Delatte, T., Schluepmann, H., Somsen, G., et al. (2013). The trehalose 6-phosphate/SnRK1 signaling pathway primes growth recovery following relief of sink limitation. Plant Physiol. 162, 1720-1732. doi: 10.1104/pp.113.220657

Price, J., Laxmi, A., St Martin, S. K., and Jang, J. C. (2004). Global transcription profiling reveals multiple sugar signal transduction mechanisms in Arabidopsis. Plant Cell 16, 2128-2150. doi: 10.1105/tpc.104.022616

Ramon, M., Rolland, F., and Sheen, J. (2008). Sugar sensing and signaling. Arabidopsis Book 6, e0117. doi: 10.1199/tab.0117

Rodrigues, A., Adamo, M., Crozet, P., Margalha, L., Confraria, A., Martinho, C., et al. (2013). ABI1 and PP2CA phosphatases are negative regulators of Snf1related Protein kinase 1 signaling in Arabidopsis. Plant Cell 25, 3871-3884. doi: $10.1105 /$ tpc.113.114066

Saeed, A., Bhagabati, N., Braisted, J., Liang, W., Sharov, V., Howe, E., et al. (2006). TM4 microarray software suite. Methods Enzymol. 411, 134-193. doi: 10.1016/s0076-6879(06)11009-5

Seo, P., Ryu, J., Kang, S., and Park, C. (2010). Modulation of sugar metabolism by an INDETERMINATE DOMAIN transcription factor contributes to photoperiodic flowering in Arabidopsis. Plant J. 65, 418-429. doi: 10.1111/j.1365-313x.2010.04432.x

Sugden, C., Crawford, R., Halford, N., and Hardie, D. (1999). Regulation of spinach SNF1-related (SnRK1) kinases by protein kinases and phosphatases is associated with phosphorylation of the $\mathrm{T}$ loop and is regulated by 5'-AMP. Plant J. 19, 433-439. doi: 10.1046/j.1365-313x.1999. 00532.x

Wahl, V., Ponnu, J., Schlereth, A., Arrivault, S., Langenecker, T., Franke, A., et al. (2013). Regulation of flowering by trehalose-6-phosphate signaling in Arabidopsis thaliana. Science 339, 704-707. doi: 10.1126/science.12 30406

Wick, A. N., Drury, D. R., Nakada, H. I., and Wolfe, J. B. (1957). Localization of the primary metabolic block produced by 2 -deoxyglucose. J. Biol. Chem. 224 , 963-969.

Winter, D., Vinegar, B., Nahal, H., Ammar, R., Wilson, G., and Provart, N. (2007). An "Electronic Fluorescent Pictograph" browser for exploring and analyzing large-scale biological data sets. PLoS ONE 2:e718. doi: 10.1371/journal.pone.0000718

Xiong, Y., McCormack, M., Li, L., Hall, Q., Xiang, C., and Sheen, J. (2013). GlucoseTOR signalling reprograms the transcriptome and activates meristems. Nature 496, 181-186. doi: 10.1038/nature12030

Yang, L., Xu, M., Koo, Y., He, J., and Poethig, R. (2013). Sugar promotes vegetative phase change in Arabidopsis thaliana by repressing the expression of MIR156A and MIR156C. Elife 2, e00260. doi: 10.7554/elife.00260

Yu, S., Cao, L., Zhou, C., Zhang, T., Lian, H., Sun, Y., et al. (2013). Sugar is an endogenous cue for juvenile-to-adult phase transition in plants. Elife 2, e00269. doi: $10.7554 /$ elife.00269

Conflict of Interest Statement: The authors declare that the research was conducted in the absence of any commercial or financial relationships that could be construed as a potential conflict of interest.

Copyright (๑) 2015 Jamsheer and Laxmi. This is an open-access article distributed under the terms of the Creative Commons Attribution License (CC BY). The use, distribution or reproduction in other forums is permitted, provided the original author(s) or licensor are credited and that the original publication in this journal is cited, in accordance with accepted academic practice. No use, distribution or reproduction is permitted which does not comply with these terms. 